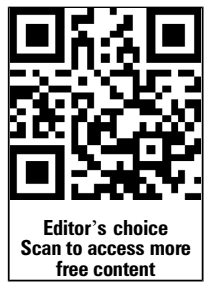

Imperial College Cerebrovascular Research Unit (ICCRU), Imperial College London, London, UK ${ }^{2}$ Department of Neurology, Mayo Clinic, Jacksonville, Florida, USA

\section{Correspondence to} Dr Pankaj Sharma, Imperial College Cerebrovascular Research Unit (ICCRU), Imperial College London, Fulham Palace Rd, London W6 8RF, UK; pankaj.sharma@imperial.ac.uk

Received 21 December 2012 Revised 19 March 2013 Accepted 22 March 2013 Published Online First 25 April 2013

To cite: Sharma P, Yadav S, Meschia JF. J Neurol

Neurosurg Psychiatry

2013;84:1302-1308.

\title{
Genetics of ischaemic stroke
}

\author{
Pankaj Sharma, ${ }^{1}$ Sunaina Yadav ${ }^{1}$ James F Meschia ${ }^{2}$
}

\section{ABSTRACT}

Recent advances in genomics and statistical computation have allowed us to begin addressing the genetic basis of stroke at a molecular level. These advances are at the cusp of making important changes to clinical practice of some monogenic forms of stroke and, in the future, are likely to revolutionise the care provided to these patients. In this review we summarise the state of knowledge in ischaemic stroke genetics particularly in the context of how a practicing clinician can best use this knowledge.

\section{INTRODUCTION}

Stroke is the third largest cause of death and disability in the developed countries, with around $25 \%$ of all strokes afflicting those less than 65 years of age. Conventional stroke risk factors such as hypertension, atrial fibrillation, cigarette smoking, diabetes mellitus and obesity are well established. However, these factors do not entirely account for the occurrence of stroke in unexposed populations and also fail to explain the incidence of stroke in select individuals within a population that is uniformly exposed to environmental risk factors. Some of this phenotypical variability has been attributed to genetic differences, with familial patterns of inheritance also lending support.

Advances in our knowledge about the workings of genes and their variants coupled with technological advances in analysing the genome along with improved bioinformatics has enabled greater understanding of the underlying molecular aetiology of ischaemic stroke. This review summarises our current state-of-knowledge in stroke genetics, particularly from a practicing clinicians' perspective.

While stroke remains principally a common sporadic disorder, our understanding of monogenic disorders has improved considerably ${ }^{1}{ }^{2}$ We begin therefore with the monogenic disorders before addressing the more common sporadic condition.

\section{MONOGENIC STROKE DISORDERS CADASIL}

Described by Joutel $e t a l,{ }^{3}$ cerebral autosomal dominant arteriopathy with subcortical infarcts and leukoencephalopathy (CADASIL) is a Mendelian form of hereditary small-vessel disease and vascular dementia. Over 100 pathogenic mutations in the $\mathrm{NOTCH} 3$ gene, an evolutionarily highly conserved transmembrane receptor protein regulating cell fate ${ }^{4}$ are known to almost always lead to an odd number of cysteine residues in one of the 34 epidermal growth factor like repeats in the extracellular domain of the Notch3 protein. These mainly missense mutations are thought to result in conformational changes of the Notch3 protein.
Mutations have predominately been identified in individuals of European descent, although cases have been found in other populations such as South Asia. ${ }^{5}$ The prevalence of CADASIL is likely underestimated, as clinical suspicion along with laboratory diagnosis is required. There are few prevalence studies, with one registry in Scotland, UK estimating prevalence rate of confirmed CADASIL cases of $1.98 / 100000 .^{6}$

Accumulation of granular osmiophilic material within the tunica media is pathophysiologically characteristic of CADASIL, ultimately leading to luminal stenosis in long penetrating arteries supplying subcortical white matter with consequent and expected reduction in cerebral blood flow. ${ }^{7}$ Nodular white matter lesions are seen on imaging and most ischaemic changes occur in the basal ganglia, periventricular white matter and temporal lobes, ${ }^{18}$ and a family with spinal cord lesions in the presence of a novel NOTCH 3 mutation has been described. ${ }^{9}$

Patients can clinically present with disorders ranging from migraine with aura $(20-40 \%$ of affected patients), ischaemic events (60-80\%), dementia, seizures, ${ }^{10}$ apathy and mood disturbances. ${ }^{11}$ Patients are usually affected with recurrent lacunar strokes by the age of 50 years despite the absence of well recognised risk factors such as hypertension. ${ }^{12}$ With advancing age these risk factors become more common and the evidence suggests that traditional risk factors accelerate disease progression. ${ }^{13}$ Though the above criteria apply to most cases, CADASIL has also been observed in cases with elderly onset, in the presence of stroke risk factors such as hypertension, and with obscure family history, ${ }^{14}$ making clinical suspicion difficult and molecular diagnosis even more important. Recently a sequencing study suggested that common variants in $\mathrm{NOTCH} 3$ gene increase the risk of age-related white matter hyperintensities in hypertensives, ${ }^{15}$ providing a nice example of Mendelian disorder informing upon a common sporadic disease.

Experimental animal models and observational human studies (clinical examination and imaging) have provided clinicians an essential understanding of the pathophysiology of CADASIL. However, genotype-phenotype correlations have been difficult to determine precisely, mainly because of the heterogeneous nature of the mutations, but some are associated with a worse prognosis. ${ }^{16}{ }^{17}$ Clinicians do however, now have a battery of methods (imaging, genetics and skin biopsy) accompanied by epidemiological data to accurately diagnose CADASIL.

Although there is no cure for CADASIL, we would argue that aggressive management of 
established risk factors ${ }^{18}$ by the clinician as well as managing symptoms (eg, acetazolamide at a dose of $125-500 \mathrm{mg} /$ daily may be an effective migraine prophylaxis ${ }^{19}$ ) are, in fact, treatment strategies that should be considered.

\section{CARASIL}

Cerebral autosomal recessive arteriopathy with subcortical infarcts and leukoencephalopathy (CARASIL) or Maeda syndrome $^{20}$ is caused by mutations in HTRA1 gene localised on ch10q encoding HtrA serine protease 1 (HTRA1) that represses signalling mediated by the transforming growth factor- $\beta$ family. ${ }^{21}$ Prevalence rates are lower than CADASIL, although it is probably more frequent than the few dozen currently reported cases, which to-date have only been described from Japan and China. ${ }^{22}$ CARASIL presents itself in the 20 s and 30 s with ischaemic stroke, dementia, premature baldness and attacks of severe lower back pain or disk herniation. Brain MRI shows diffuse white matter changes and multiple lacunar infarctions in the basal ganglia and thalamus. ${ }^{22}$ Histopathologically, arteriosclerosis is seen in the penetrating arteries in the absence of granular osmiophilic or amyloid material. ${ }^{23}$ Patients with CARASIL are also less likely to have migraines and exhibit psychiatric disorders, such as euphoria and emotional liability. ${ }^{1}$

\section{FABRY'S DISEASE}

Fabry's disease is a congenital metabolic disorder caused by deficient activity of $\alpha$-galactosidase A ( $\alpha$-gal), resulting in a progressive accumulation of globotriaosylceramide and related glycosphingolipids within vascular endothelial cells, myocardial cells and neurons. ${ }^{24}$ The prevalence of Fabry's disease in patients with stroke is variable but has been reported to be as high as $4.9 \%{ }^{25}$ to $6.9 \%$ with a median age of onset of 39 years in men, ${ }^{26}$ otherwise the incidence is reported as 1 per 3100 individuals. ${ }^{27}$ Although an $\mathrm{X}$ linked lysosomal storage disorder, female carriers can develop symptoms suggestive of Fabry's disease $^{28}$ with symptoms appearing comparatively later than in men, at a median age of 45.7 years. $^{26}$

Neurological symptoms are wide-ranging with the most common clinical presentation being peripheral neuropathy presenting as burning pain in the extremities and gastrointestinal symptoms. Fabry's disease should be considered in patients with small-fibre neuropathy of unknown cause. ${ }^{29}$ Ischaemic stroke and transient ischaemic attack (TIA) are also frequent presentations in Fabry's disease, with abnormalities of the vertebral and basilar arteries particularly common. ${ }^{30}$ MRI classically shows high intensity lesions in the pulvinar and torturous and dilated large vessels (dolichoectasia). The well recognised pulvinar sign (hyperintensity in the pulvinar nucleus of the thalami) as well as increased basilar artery diameter are highly characteristic of this disease, ${ }^{31}$ although this sign has also been reported in Wernicke's and paraneoplastic encephalopathy, vacuolar leucoencephalopathy in association with coeliac disease as well as sporadic and variant Creutzfeldt-Jakob disease. Fabry's symptoms are not specific and diagnosis through clinical suspicion and imaging is insufficient to confirm diagnosis. Measuring the $\alpha$-gal activity in leukocytes and plasma and sequencing the $\alpha$-GAL gene for the over 400 known pathological mutations confirms the presence of Fabry's. ${ }^{1}$

Bi-weekly recombinant $\alpha$-gal enzyme replacement therapy at a dose of $1 \mathrm{mg} / \mathrm{kg}$ body weight has shown benefits for heart, kidney and neuropathic pain, especially if patients are treated early in their disease; ${ }^{32}{ }^{33}$ however, the risk of stroke remains substantial and management of conventional stroke risk factors is important as well as early augmentation of enzyme replacement therapy. ${ }^{34} 35$

\section{MELAS}

Mitochondrial myopathy, encephalopathy, lactic acidosis and stroke-like episodes (MELAS) is one of the most clinically prevalent and commonly encountered genetic disorder, $80 \%$ of which is accounted for by maternally transmitted mitochondrial tRNA (Leu) A3243G mutations. ${ }^{36}$ Another $10 \%$ of patients carry the T3271C mutation. The prevalence of MELAS varies from 7.9/100 000 in England to 236/100 000 in Australia ${ }^{37}$ with an age of onset ranging from 2 years to 20 years. Typically, symptoms manifest in childhood including migraines, seizures, anorexia and vomiting. ${ }^{2}$

Stroke-like episodes occur in MELAS, but the aetiology of those strokes has been given several explanations ${ }^{38}$ ranging from vasogenic oedema, ${ }^{39}$ cytopathic toxicity and hyperperfusion. ${ }^{40} \mathrm{MRI}$ and MR spectroscopy show lesions that do not follow recognised arterial territory distribution and have a tendency to affect the posterior (temporal, occipital and parietal) aspects of the brain. ${ }^{38}$

The diagnosis of MELAS can be confirmed with a skeletal muscle biopsy, which stains strongly positive with succinate dehydrogenase and which shows ragged morphology of skeletal fibres on modified Gomori trichrome. ${ }^{38}$ Most patients also have increased lactate/pyruvate ratios in serum and cerebral spinal fluid (CSF). ${ }^{41}$

Most therapeutic strategies to treat MELAS use supplements and enzyme cofactors that enhance mitochondrial metabolism and activity of the respiratory chain. ${ }^{42}$ Small studies (mostly case reports) have demonstrated the effects of coenzyme Q10 and its synthetic analogue, idebenone, to have an effect on decreasing lactic acid levels ${ }^{43}$ and reducing stroke-like episodes in patients with MELAS. $^{44}$ Dichloroacetate, an inorganic enzyme activator, which reduces lactic acid levels was tested on patients with MELAS without showing any health benefits and discontinued due to neurotoxicity. ${ }^{45} \mathrm{~L}$-arginine has also been tested on patients with MELAS with stroke-like episodes and a significant reduction in frequency of events and ischaemic injury was observed. ${ }^{46}$ Other potential therapies include oral administration of vitamins (B complex, E and C), steroids, coenzyme $\mathrm{Q} 10$, idebenone and L-arginine ${ }^{2}$; however their clinical benefits have yet to be determined.

\section{OTHER MONOGENIC DISEASES}

Other single-gene diseases ${ }^{1}{ }^{2}$ have also been associated with (usually childhood) ischaemic stroke including sickle cell ${ }^{47}$ and homocystinuria. ${ }^{48}$

\section{WHITE MATTER DISEASE}

White matter lesions are commonly seen on T2-weighted MRIs in up to $70 \%$ of elderly patients, ${ }^{49}$ the lesion numbers and extent increasing with age. Although several factors are likely to account for the presence of these lesions, including hypertension, atherosclerosis and cigarette smoking, the final common pathway is likely to be chronic ischaemia, ${ }^{50}$ among other factors. The presence of these white matter lesions is of prognostic importance, as they increase the likelihood of stroke $e^{51}$ and cognitive decline. ${ }^{52}$

White matter disease has been shown to have a heritable basis with estimates varying around $0.66-0.72^{53}$ depending on the whether the lesions are subcortical or global-the latter having greater heritability. With such high heritability estimates, numerous investigators have explored the role of several candidate genes, but with few convincing replications; while genome-wide 
association models without an a priori hypothesis have not fared much better. ${ }^{54}$ Notwithstanding the unimpressive results thus far, the epidemiological evidence for heritability does suggest that there are susceptibility loci to be found. That view has seen recent support when, following better phenotypical classification of white matter disease and a greater number of subjects recruited to improve power, a locus on $17 \mathrm{q} 25$ associated with white matter disease (WMD) was identified in a population of European descent. ${ }^{55}$

\section{SPORADIC ISCHAEMIC STROKE}

While monogenic disorders are highly penetrant, they remain relatively rare as a cause of stroke. Established causes of cerebrovascular disease such as hypertension and diabetes of course are extremely important, ${ }^{56}$ but these environmental determinants are likely influenced by inherited risk factors and a considerable wealth of data supports a genetic basis for stroke.

\section{HERITABILITY OF SPORADIC STROKE}

Animal studies have suggested an underlying genetic basis for stroke. ${ }^{57}$ In humans, twin studies have shown that a positive family history is a risk factor for stroke (OR 1.65; 95\% CI 1.2 to 2.3) and monozygotic twins are more likely to be concordant than dizygotic twins (OR, 1.65; 95\% CI 1.2 to 2.3$).{ }^{58}$ Further, stroke tends to run in families, leading researchers to suspect an inherited component (although it would not be surprising that a common disease like stroke is often found within families as family member's age). Most family ${ }^{59} 60$ and twin studies ${ }^{61} 62$ suggest the genetic liability is greater in patients aged younger than 70 years $^{58} 63$ and varies with stroke subtype. ${ }^{64}$ Case-control studies suggest a $76 \%$ increase in the risk of ischaemic stroke in the presence of a family history of stroke, ${ }^{58}$ although not all reports have demonstrated a positive relationship with family history ${ }^{65}$ possibly due to confounding factors such as blood pressure. ${ }^{66}$ Heritability of stroke has also recently been estimated using data derived from genome-wide association studies (GWAS). Genome-wide complex trait analysis-a tool that allows researchers to estimate heritability of a complex phenotypical trait such as stroke using genome wide association single nucleotide polymorphism (SNP) data on unrelated individuals-estimates variance explained by all SNPs entered against a phenotypical trait rather than individual SNPs. ${ }^{67}$ Using this methodology, heritability estimate for all ischaemic strokes was $37.9 \%$ but varied considerably by subtype with the greatest associated with large vessel $(40.3 \%)$ and cardioembolic disease $(32.6 \%)$ and lowest for small vessel disease $(16.1 \%){ }^{67}$ The phenotypical heterogeneity between stroke subtypes and differences in aetiology could potentially explain the differences in heritability estimates.

\section{GENES FOR STROKE}

In a comprehensive meta-analysis of 187 candidate genes in 37481 ischaemic stroke cases and 95322 controls, five genes were identified that robustly associated with stroke susceptibility Factor V ArgGln506 (OR 1.31), ACE/ID (OR 1.15), MTHFR C677T (OR 1.26), Prothrombin G20210A (OR 1.60), PAI-1 5G (OR 1.11) and Glycoprotein IIIa Leu33Pro (OR 1.24). ${ }^{68}$ With the exception of MTHFR, which is a metabolic enzyme controlling plasma homocysteine levels, the other genes belong to the blood coagulation system, and mutations in these genes may predispose for ischaemic or haemorrhagic stroke, depending on the type of mutation. Experimental studies have yet to establish the exact role for these genes in human stroke. While the effect sizes per risk allele are small, the sum of the population-attributable risks across all associations was $\sim 30 \%$, which, given the relative frequency of stroke, translates to a large clinically observed effect (although publication bias probably inflated the estimate of risk). Conversely, although the monogenic disorders confer a high individual relative risk, they contribute very little to population stroke due to their rarity. ${ }^{68}$

Although the gene variants identified in literature based meta-analysis have not been convincingly replicated in GWAS studies, ${ }^{67}$ this does not discount the validity of those findings. For example, MTHFR is a well studied genetic risk variant of ischaemic stroke and elevated levels of its intermediate phenotype, homocysteine, have been causally associated with the increased risk of stroke. ${ }^{68}{ }^{69}$ In addition, coverage of the human genome with genome-wide studies is neither equal across regions of the genome nor comprehensive. Many previously associated candidate genes such as $A C E$ are not adequately covered by the currently commercially available GWAS platforms.

The choice of the candidate gene investigated tended to be based on the investigators 'best guess' or a local research interest in that biological pathway or candidates derived from presumed related vascular conditions, most commonly from the cardiology literature. This is not surprising as the pathophysiology of the stroke and heart disease has appeared similar, and so researchers took their best guess from a related field. However, these predictions have only occasionally lived up to expectations, ${ }^{70}$ with some candidates appearing to be organ-specific rather than pathophysiologyspecific. ${ }^{68}$ The organ specific association of genetic variants could be partly explained through the effect of confounding risk factors such as diabetes, hypertension and elevated plasma cholesterol levels, which demonstrate arterial specificity; for example, cholesterol is mainly important for coronary artery disease (CAD) while hypertension mainly for ischaemic stroke. ${ }^{7172}$

Candidate gene approaches have been helpful in indicating the likely risk ratios that a risk allele would imply. While the odds associated with these SNPs are varied due to small sample sizes, meta-analyses of these studies have pooled data and provided robust results with ORs ranging between 1.2 and 2.6 depending on the gene of interest. ${ }^{73}$ These ORs have broadly held true across different ethnic populations with few exceptions, ${ }^{74}$ although the evidence base in non-Europeans is considerably smaller, and the lack of research here should be a cause for concern for investigators. However, the emergence of genome-wide approaches that test anonymous loci unburdened by any a priori hypothesis (see below) have presented investigators with an alternative method to test the productivity of the candidate-based approach. ${ }^{67}$ Using highly characterised stroke cases from the Wellcome Trust Case Control Consortium that have been subjected to a whole-genome search, investigators determined that of the 32 previously reported candidate genes associated with ischaemic stroke, 4 were still associated following multiple correction analysis but not when more stringent criteria were applied. $^{67}$ Conversely, with the three GWAS-associated genes (see below) PHACTR1 in large vessel disease, PITX2 and ZFHX3, cardiac embolic stroke held true.

These studies have substantiated our contention that stroke is a complex disorder with low locus-specific ORs similar to other multifactorial diseases such as hypertension and coronary artery disease $^{75} 76$ (table 1).

\section{CAUSAL RELATIONSHIPS}

When conducted in large appropriately phenotyped and matched populations, association studies have been successful in identifying stroke susceptibility genes and have stood the test of time. ${ }^{77}$ While such observational studies help us understand the 
Table 1 OR $(95 \% \mathrm{Cl})$ with effect differences between heart disease and ischaemic stroke (adapted from ref. ${ }^{68}$ )

\begin{tabular}{|c|c|c|c|c|}
\hline Gene & Polymorphism & MI/IHD & IS & Effect \\
\hline Factor V Leiden & Arg506Gln & 1.17 (1.08 to 1.28$)$ & 1.25 (1.08 to 1.45$)$ & $\mathrm{MI}=\mathrm{IS}$ \\
\hline ACE & I/D & 1.21 (1.11 to 1.32$)$ & 1.15 (1.06 to 1.25$)$ & $\mathrm{MI}=\mathrm{IS}$ \\
\hline MTHFR & С677T & 1.16 (1.05 to 1.28$)$ & 1.27 (1.08 to 1.48$)$ & $\mathrm{MI}=\mathrm{IS}$ \\
\hline Prothrombin & G20210A & 1.25 (1.05 to 1.50$)$ & 1.62 (1.29 to 2.04$)$ & $\mid \mathrm{S}>\mathrm{MI}$ \\
\hline Glycoprotein IIla & Leu33Pro & $1.02(0.96$ to 1.07$)$ & 1.20 (1.08 to 1.34$)$ & IS only \\
\hline PAI-1 & $4 \mathrm{G} / 5 \mathrm{G}$ & $1.06(1.02$ to 1.10$)$ & $0.90(0.82$ to 0.99$)$ & $>\mathrm{Ml}<\mathrm{IS} *$ \\
\hline Angiotensinogen & M235T & $1.11(1.03$ to 1.19$)$ & 0.96 (0.87 to 1.02$)$ & $>\mathrm{Ml}<\mathrm{IS}^{*}$ \\
\hline
\end{tabular}

genetic underpinning of risk, they are silent on whether there exists a causal relationship between the risk variants and disease.

Mendelian randomisation has been used in an attempt to support a causal relationship between risk genotype and phenotype. ${ }^{78}$ The method is predicated firstly on an observational association between a biochemical factor and disease, and secondarily on whether concordant levels of risk occur for a genotype that simulates the biochemical factor. ${ }^{78}$ As randomisation to the genotype of interest occurs at gamete formation, the arm to which the subject is assigned is not burdened by any bias. Results from Mendelian randomisation strategies are therefore more likely to support causation.

This strategy was first successfully approached in human stroke with MTHFR and homocysteine ${ }^{79}$ and subsequently has demonstrated causal relationships to ischaemic stroke for Factor V Leiden, ACE and Prothrombin G20210A. ${ }^{68}$ While causal relationships to stroke have been demonstrated for homocysteine, randomised clinical trials have failed to demonstrate a clinical benefit in reducing homocysteine levels with vitamin supplementation. ${ }^{80}$ However, there may be a variety of factors for this result, including the chosen dose of the treatment regimen to the length of follow-up in the trial perhaps being too short to reverse long-term effects of homocysteine. Moreover, the effect of the MTHFR variant on homocysteine concentration is greatest in regions of the world (eg, Asia) where there is no government policy of folate fortification. ${ }^{81}$ This contrasts with many Western countries (America, Australia, New Zealand, etc) where a programme of folate fortification leads to higher baseline levels and, paradoxically, is where most of the (negative) folate reduction clinical trials have been conducted. Trials of lowering homocysteine and determining stroke risk may better be served in global regions of endemic low folate. However, this position has not always found universal support with all forms of cardiovascular disease. ${ }^{82}$

There are several limitations of the Mendelian randomisation method. Basing a causal relationship on an associated locus could be fallacious as the variant could be in linkage disequilibrium with the actual causative variant that was not assessed. Another major limitation could be the inability to calculate a strong genetic instrumental variable to assess the causal effect of a phenotype on an outcome. ${ }^{83}$ In the case of homocysteine, plasma levels of the biomarker can also be affected by other genes, such as MTR and MTRR, and therefore the choice of the correct instrumental variable is essential to decipher the appropriate causal relationship.

\section{GENOME-WIDE ASSOCIATION STUDIES}

With completion of the Human Genome Project in 2003, scientists identified regions of variation between individuals, the most common form of which is the SNP. The human genome is believed to consist of over 10 million SNPs and, with the efforts of the International HapMap project, three million SNPs have been characterised. Information provided by HapMap has enabled the development of commercially available genotyping microarrays and heralded the era of the GWAS. As technology used to unravel the genetic basis of disease has advanced, our ability to rapidly and relatively inexpensively search for susceptibility loci without a priori hypotheses has dramatically improved. Individual candidate gene studies have predominantly been replaced by whole-genome screening, which has been successfully conducted in a variety of disorders. ${ }^{84} 85$

GWAS on stroke were few and far between till 2003 when Gretarsdottir et $a l^{86}$ identified phosphodiesterase 4D to be significantly associated with risk of ischaemic stroke in an Icelandic population (DeCODE). However several attempts to replicate these findings failed, ${ }^{87} 88$ while some studies reported conflicting results. ${ }^{89} 90$ These discrepancies were attributed to possible problems in study design, that is, pooling of carotid embolism and large vessel disease strokes in order to identify the risk association with phosphodiesterase 4D, which we now know are subtype specific. ${ }^{91}$ (Lack of independent replication and limited experimental validation of results using B cell lines was also suggested to be a major limitation of the original study ${ }^{92}$ ). Several other GWAS followed, but no single locus was identified at a genome-wide level of significance defined as $\mathrm{p}<10^{-8}$.

The Wellcome Trust Case Control Consortium 2 and the International Stroke Genetics Consortium performed a GWAS involving 3548 cases of ischaemic stroke with replication of potential signals in 5859 additional cases. ${ }^{93}$ The study demonstrated, as others had done previously, ${ }^{94} 95$ associations for cardioembolic stroke near PITX2 and ZFHX3, which are known risk loci for atrial fibrillation. ${ }^{96}$ The study also confirmed the association for large vessel stroke and a 9p21 locus. A novel finding was an association for large vessel stroke within HDAC9 on chromosome 7p21.1 (OR 1.42). The recent METASTROKE ${ }^{97}$ meta-analysis, which included 15 stroke cohorts comprising $\sim 12000$ cases and $\sim 60000$ controls, failed to identify any new genetic risk variants and only validated previous findings of genes PITX2, ZFHX3 and HDAC9. All loci exhibited heterogeneous effect across subtypes, supporting distinct genetic architectures for each subtype.

Several confounding factors can lead to the failure of replication of a GWAS-discovery SNP. Errors in genotyping, quality control and choice of analytical methods can lead to false positive results as has been implicated for the DeCODE study. ${ }^{92}$ The European population is genetically stratified ${ }^{98}$ and an admixture of populations with different ancestry can also lead to inflated statistics. Results from large GWAS have implied that dissecting out the susceptibility genes for stroke needs to consider its 
subtypes as different entities. This should not be surprising, as 'stroke' is a clinical syndrome encompassing any sudden focal neurological deficit from a vascular aetiology. Investigators have therefore increasingly begun to analyse stroke genetic datasets by subtype. In a very recent GWAS, the evidence for a genetic influence on all-cause ischaemic stroke became more compelling with large artery stroke subtype. ${ }^{91}$ This new finding on $6 \mathrm{p} 21.1$ was also replicated using other independent datasets. ${ }^{91}$

Using incident cases from the Rotterdam study as the discovery cohort, a novel genetic locus on chromosome $\mathrm{X}$ near the androgen receptor gene was found to associate with vascular dementia as defined by the National Institute of Neurological Disorders and Stroke and Association (NINDS-AIREN) criteria, and the SNP rs12007229 association replicated in two independent cohorts. ${ }^{99}$ Similar effect sizes were noted for men and women, but independent confirmation of this finding is warranted. A further GWAS has identified association between cerebral white matter lesion volume and a locus on chromosome $17 \mathrm{q} 25 .^{55}$ A recent meta-analysis of all published data supported the association $\left(\mathrm{rs} 3744028 ; \mathrm{p}=5.3 \times 10^{-17}\right){ }^{100}$

Several other GWAS have been conducted in stroke mostly in those of European descent, but also a few of Asian ancestry ${ }^{101-103}$ (table 2). The ORs range from 1.00 to 1.85 , confirming that the effect sizes are small but the attributable risks could be large given the common nature of this condition. Many studies however have failed to replicate their findings. ${ }^{108} 109106$ Noting that some ischaemic stroke has a maternal heritability, a GWAS of common mitochondrial sequence variants failed to find a genome significance threshold, although this study was underpowered for GWAS. ${ }^{110}$ Some investigators have undertaken GWAS on surrogate markers such as white matter hyperintensities intermediate phenotypes ${ }^{111}$ or intermediate phenotypes such as intima-media thinkness. ${ }^{112}$

The fact that most of these studies have been conducted in individuals of European descent is a blot on the stroke genetic landscape with very little comparative data available in other populations, although establishment of high quality biobanks is now underway (eg, South Asia and Middle East) and is expected to deliver interesting results. ${ }^{113} 114$

A recent pilot study identified rare exonic variants to be associated with stroke suggesting that coding variations in the human genome need to be closely examined. ${ }^{115}$ The genetic architecture of stroke is complex and is likely to include non-SNP variations as well. Large structural genomic variations such as copy number variations (CNVs) have been known to play a role in monogenic disorders; however their role in complex traits such as stroke is less clear. GWA of copy number variations associated with ischaemic stroke has not identified any unique structural genomic variations that may contribute to the risk for stroke. ${ }^{116}$ Smaller candidate gene based studies have provided some evidence of a unique genomic structure in patients with ischaemic stroke, ${ }^{117}$ however large well powered studies have failed to do the same. ${ }^{118}$

There are several ongoing challenges in applying GWAS data to everyday clinical practices and individual patient care, since available directives are mostly speculative. The most widely held belief is that based on genetic information and clinical phenotypes of an individual, tailor made treatment plans can be designed by clinicians. Genetic testing kits can help clinicians test their patients for risk variants and provide them with information about risk factors that can act as confounders for the disease. With the addition of genotype information to clinical phenotype based predictive disease models, the prognostic ability can be improved. Possible pharmacogenetic effects may also be predicted based on the knowledge of the patients' genotype. However, the complexity of a polygenic syndrome such as stroke is immense and, with the exception of certain highly penetrant monogenic forms of stroke such as CADASIL, the prognosis of stroke is difficult based on common genetic variants that occur in only $1-5 \%$ of the general population and have modest to small effect sizes. Even though results from the genome wide association testing of stroke are promising they currently have no value in predicting risk and treatment.

\section{CONCLUSION}

The understanding of stroke genetics has made significant progress in the past few years and has provided clinicians with in-depth knowledge of the molecular underpinnings of the disease that can be useful in improving patient care. However, unlike many of the other conditions being tackled, stroke has several orders of complexity that need to be managed. As an age-related condition, family clusters are difficult to collect and dissecting out the genetic component to its aetiology from environmental determinants is that much harder. Moreover, stroke is a heterogeneous condition comprised of a number of subtypes that are likely to have different genetic aetiologies. Only recently are these subtypes being accurately and reliably characterised to allow a more refined phenotype to be explored with a greater degree of focus. Notwithstanding these unique issues, international collaborations have been successful in a variety of stroke subtypes and the results of more of these collaborations in different ethnic populations are eagerly awaited.

Table 2 Genome-wide association studies (GWAS) in stroke

\begin{tabular}{|c|c|c|c|c|c|c|}
\hline Chr & Gene & Subtype & RA & RAF & OR (95\% Cl) & $\mathrm{p}$ Value \\
\hline $7 p 21.1$ & HDAC9 9397 & LVD & $A$ & 0.09 & 1.39 (1.27 to 1.53$)$ & $2.03 \times 10^{-12}$ \\
\hline $4 q 25$ & PITX2 $2^{95} 97$ & $\mathrm{CE}$ & A & 0.19 & 1.36 (1.27 to 1.47$)$ & $2.8 \times 10^{-16}$ \\
\hline $9 p 21.3$ & CDKN2A/B ${ }^{97} 104105$ & LVD & G & 0.51 & 1.17 (1.09 to 1.25$)$ & $2.93 \times 10^{-5}$ \\
\hline $12 p 13.33$ & NINJ2 $2^{106}$ & All & $A$ & 0.23 & 1.41 (1.27 to 1.56$)$ & $2.3 \times 10^{-10}$ \\
\hline $16 q 22.3$ & ZFHX3 ${ }^{94} 97$ & CE & G & 0.17 & 1.25 (1.15 to 1.35$)$ & $2.28 \times 10^{-8}$ \\
\hline $11 q 12$ & AGTRL1 ${ }^{103}$ & All & G & - & 1.3 (1.14 to 1.47$)$ & $6.66 \times 10^{-5}$ \\
\hline- & CELSR $1^{102}$ & All & G & - & 1.85 (1.29 to 2.61$)$ & $1 \times 10^{-4}$ \\
\hline $5 q 12$ & PDE4D ${ }^{86}$ & CE and cryptogenic & & 0.16 & - & $1.5 \times 10^{-6}$ \\
\hline $13 q 12-13$ & ALOX5AP 107 & All & & 0.15 & 1.67 & $9.5 \times 10^{-5}$ \\
\hline- & PRKCH $^{101}$ & SVD & & - & 1.4 & $5.1 \times 10^{-7}$ \\
\hline $6 p 21.1$ & rs556621 $1^{91}$ & LVD & & & 1.21 & $4.7 \times 10^{-8}$ \\
\hline
\end{tabular}

Chr, chromosome; CE, carotid embolism; LVD, large vessel disease; $p$, probability value; RA, risk allele; RAF, risk allele frequency; SVD, small vessel disease. 
Acknowledgements PS is supported by a Department of Health (UK) senior fellowship. SY is supported by the UK-India Education and Research Initiative (UKIERI) from the British Council. JFM is supported by the NINDS (USA) through a grant to the Stroke Genetics Network (SiGN; grant number NINDS 1 U01 NS069208).

Contributors PS conceived the idea and drafted the original version. SY and JFM critically revised the manuscript with important intellectual content. All authors approved the final version.

\section{Competing interests None.}

Provenance and peer review Not commissioned; externally peer reviewed.

\section{REFERENCES}

1 Muqtadar $H$, Testai FD. Single gene disorders associated with stroke: a review and update on treatment options. Curr Treat Options Cardiovasc Med 2012;14:288-97.

2 Della-Morte D, Guadagni F, Palmirotta R, et al. Genetics of ischemic stroke, stroke-related risk factors, stroke precursors and treatments. Pharmacogenomics 2012;13:595-613.

3 Joutel A, Corpechot C, Ducros A, et al. Notch3 mutations in CADASIL, a hereditary adult-onset condition causing stroke and dementia. Nature 1996:383:707-10.

4 Artavanis-Tsakonas S, Rand MD, Lake RJ. Notch signaling: cell fate control and signal integration in development. Science 1999;284:770-6.

5 Yadav S, Bentley P, Srivastava P, et al. I Stroke Cerebrovasc Dis 2013;22: 28-31.

6 Razvi SS, Davidson R, Bone I, et al. The prevalence of cerebral autosomal dominant arteriopathy with subcortical infarcts and leucoencephalopathy (CADASIL) in the west of Scotland. J Neurol Neurosurg Psychiatry 2005;76:739-41.

7 Chabriat H, Pappata S, Ostergaard L, et al. Cerebral hemodynamics in CADASIL before and after acetazolamide challenge assessed with MRI bolus tracking. Stroke 2000;31:1904-12.

8 O'Sullivan M, Jarosz JM, Martin RJ, et al. MRI hyperintensities of the temporal lobe and external capsule in patients with CADASIL. Neurology 2001;56:628-34.

9 Bentley P, Wang T, Malik 0, et al. CADASIL with cord involvement associated with a novel and atypical NOTCH3 mutation. J Neurol Neurosurg Psychiatry 2011;82:855-60.

10 Sharma P, Wang T, Brown MJ, et al. Fits and strokes. Lancet 2001;358:120.

11 Herve D, Chabriat H. Cadasil. J Geriatr Psychiatry Neurol 2010;23:269-76.

12 Ayata C. CADASIL: experimental insights from animal models. Stroke 2010;41: S129-34.

13 Adib-Samii P, Brice G, Martin RJ, et al. Clinical spectrum of CADASIL and the effect of cardiovascular risk factors on phenotype: study in 200 consecutively recruited individuals. Stroke 2010;41:630-4.

14 Mizuno T. [Diagnosis, pathomechanism and treatment of CADASIL]. Rinsho Shinkeigaku 2012;52:303-13.

15 Schmidt $H$, Zeginigg $M$, Wiltgen $M$, et al. Genetic variants of the NOTCH3 gene in the elderly and magnetic resonance imaging correlates of age-related cerebral small vessel disease. Brain 2011;134:3384-97.

16 Monet-Lepretre M, Bardot B, Lemaire B, et al. Distinct phenotypic and functional features of CADASIL mutations in the Notch3 ligand binding domain. Brain 2009:132:1601-12.

17 Opherk C, Peters N, Herzog J, et al. Long-term prognosis and causes of death in CADASIL: a retrospective study in 411 patients. Brain 2004;127:2533-9.

18 del Rio-Espinola A, Mendioroz M, Domingues-Montanari S, et al. CADASIL management or what to do when there is little one can do. Expert Rev Neurother 2009:9:197-210.

19 Donnini I, Nannucci S, Valenti R, et al. Acetazolamide for the prophylaxis of migraine in CADASIL: a preliminary experience. J Headache Pain 2012;13:299-302.

20 Fukutake T, Hirayama K. Familial young-adult-onset arteriosclerotic leukoencephalopathy with alopecia and lumbago without arterial hypertension. Eur Neurol 1995:35:69-79.

21 Hara K, Shiga A, Fukutake T, et al. Association of HTRA1 mutations and familial ischemic cerebral small-vessel disease. N Engl J Med 2009;360:1729-39.

22 Fukutake T. Cerebral autosomal recessive arteriopathy with subcortical infarcts and leukoencephalopathy (CARASIL): from discovery to gene identification. J Stroke Cerebrovasc Dis 2011;20:85-93.

23 Arima K, Yanagawa S, Ito N, et al. Cerebral arterial pathology of CADASIL and CARASIL (Maeda syndrome). Neuropathology 2003;23:327-34.

24 Toyooka K. Fabry disease. Curr Opin Neurol 2011;24:463-8.

25 Rolfs $A$, Bottcher $T$, Zschiesche $M$, et al. Prevalence of Fabry disease in patients with cryptogenic stroke: a prospective study. Lancet 2005;366:1794-6.

26 Sims K, Politei J, Banikazemi M, et al. Stroke in Fabry disease frequently occurs before diagnosis and in the absence of other clinical events: natural history data from the Fabry Registry. Stroke 2009:40:788-94.

27 Spada M, Pagliardini S, Yasuda M, et al. High incidence of later-onset fabry disease revealed by newborn screening. Am J Hum Genet 2006:79:31-40.
28 Wilcox WR, Oliveira JP, Hopkin RJ, et al. Females with Fabry disease frequently have major organ involvement: lessons from the Fabry Registry. Mol Genet Metab 2008:93:112-28.

29 Tanislav C, Kaps M, Rolfs A, et al. Frequency of Fabry disease in patients with small-fibre neuropathy of unknown aetiology: a pilot study. Eur J Neurol 2011;18:631-6.

30 Mitsias P, Levine SR. Cerebrovascular complications of Fabry's disease. Ann Neurol 1996:40:8-17.

31 Fellgiebel A, Keller I, Marin D, et al. Diagnostic utility of different MRI and MR angiography measures in Fabry disease. Neurology 2009;72:63-8.

32 Mehta A, Beck M, Elliott $P$, et al. Enzyme replacement therapy with agalsidase alfa in patients with Fabry's disease: an analysis of registry data. Lancet 2009;374:1986-96.

33 Lidove 0, West ML, Pintos-Morell G, et al. Effects of enzyme replacement therapy in Fabry disease-a comprehensive review of the medical literature. Genet Med 2010;12:668-79.

34 Furie KL, Kasner SE, Adams RJ, et al. Guidelines for the prevention of stroke in patients with stroke or transient ischemic attack: a guideline for healthcare professionals from the american heart association/american stroke association. Stroke 2011;42:227-76.

35 Germain DP. Fabry disease: the need to stratify patient populations to better understand the outcome of enzyme replacement therapy. Clin Ther 2007;29 (Suppl A):S17-18.

36 Goto Y, Horai S, Matsuoka T, et al. Mitochondrial myopathy, encephalopathy, lactic acidosis, and stroke-like episodes (MELAS): a correlative study of the clinical features and mitochondrial DNA mutation. Neurology 1992;42:545-50.

37 Manwaring N, Jones MM, Wang JJ, et al. Population prevalence of the MELAS A3243G mutation. Mitochondrion 2007:7:230-3.

38 Testai FD, Gorelick PB. Inherited metabolic disorders and stroke part 1: Fabry disease and mitochondrial myopathy, encephalopathy, lactic acidosis, and strokelike episodes. Arch Neurol 2010;67:19-24

39 Yoneda $M$, Maeda $M$, Kimura $H$, et al. Vasogenic edema on MELAS: a serial study with diffusion-weighted MR imaging. Neurology 1999;53:2182-4.

40 Ito $\mathrm{H}$, Mori K, Harada M, et al. Serial brain imaging analysis of stroke-like episodes in MELAS. Brain Dev 2008;30:483-8.

41 Yamada K, Toribe Y, Yanagihara K, et al. Diagnostic accuracy of blood and CSF lactate in identifying children with mitochondrial diseases affecting the central nervous system. Brain Dev 2012:34:92-7.

42 Santa KM. Treatment options for mitochondrial myopathy, encephalopathy, lactic acidosis, and stroke-like episodes (MELAS) syndrome. Pharmacotherapy 2010:30:1179-96.

43 Rodriguez MC, MacDonald JR, Mahoney DJ, et al. Beneficial effects of creatine, CoQ10, and lipoic acid in mitochondrial disorders. Muscle Nerve 2007;35:235-42.

44 Napolitano A, Salvetti S, Vista M, et al. Long-term treatment with idebenone and riboflavin in a patient with MELAS. Neuro/ Sci 2000;21:S981-2.

45 Kaufmann P, Engelstad K, Wei Y, et al. Dichloroacetate causes toxic neuropathy in MELAS: a randomized, controlled clinical trial. Neurology 2006;66:324-30.

46 Koga Y, Povalko N, Nishioka J, et al. MELAS and L-arginine therapy: pathophysiology of stroke-like episodes. Ann N Y Acad Sci 2010;1201:104-10.

47 Switzer JA, Hess DC, Nichols FT, et al. Pathophysiology and treatment of stroke in sickle-cell disease: present and future. Lancet Neurol 2006;5:501-12.

48 Kelly PJ, Furie KL, Kistler JP, et al. Stroke in young patients with hyperhomocysteinemia due to cystathionine beta-synthase deficiency. Neurology 2003;60:275-9

49 Schmidt R, Enzinger C, Ropele S, et al. Progression of cerebral white matter lesions: 6-year results of the Austrian Stroke Prevention Study. Lancet 2003;361:2046-8.

50 Rost N. White Matter Disease. In: Sharma P, Meschia JF, eds. Stroke genetics. London: Springer, 2013:171-88.

51 Kuller LH, Longstreth WT Jr, Arnold AM, et al. White matter hyperintensity on cranial magnetic resonance imaging: a predictor of stroke. Stroke 2004;35:1821-5.

52 de Groot JC, de Leeuw FE, Oudkerk M, et al. Cerebral white matter lesions and subjective cognitive dysfunction: the Rotterdam Scan Study. Neurology 2001;56:1539-45.

53 Carmelli D, DeCarli C, Swan GE, et al. Evidence for genetic variance in white matter hyperintensity volume in normal elderly male twins. Stroke 1998;29:1177-81.

54 Turner ST, Fornage M, Jack CR Jr, et al. Genomic susceptibility loci for brain atrophy in hypertensive sibships from the GENOA study. Hypertension 2005:45:793-8

55 Fornage M, Debette S, Bis JC, et al. Genome-wide association studies of cerebral white matter lesion burden: the CHARGE consortium. Ann Neurol 2011;69:928-39.

56 O'Donnell MJ, Xavier D, Liu L, et al. Risk factors for ischaemic and intracerebral haemorrhagic stroke in 22 countries (the INTERSTROKE study): a case-control study. Lancet 2010;376:112-23.

57 Jeffs B, Clark JS, Anderson NH, et al. Sensitivity to cerebral ischaemic insult in a rat model of stroke is determined by a single genetic locus. Nat Genet 1997:16:364-7. 
58 Flossmann E, Schulz UG, Rothwell PM. Systematic review of methods and results of studies of the genetic epidemiology of ischemic stroke. Stroke 2004;35:212-27.

59 Jousilahti P, Rastenyte D, Tuomilehto J, et al. Parental history of cardiovascular disease and risk of stroke. A prospective follow-up of 14371 middle-aged men and women in Finland. Stroke 1997;28:1361-6.

60 Liao D, Myers R, Hunt $S$, et al. Familial history of stroke and stroke risk. The Family Heart Study. Stroke 1997;28:1908-12.

61 Bak S, Gaist D, Sindrup SH, et al. Genetic liability in stroke: a long-term follow-up study of Danish twins. Stroke 2002;33:769-74.

62 Brass LM, Isaacsohn JL, Merikangas KR, et al. A study of twins and stroke. Stroke 1992;23:221-3

63 Jood K, Ladenvall P, Tjarnlund-Wolf A, et al. Fibrinolytic gene polymorphism and ischemic stroke. Stroke 2005;36:2077-81.

64 Schulz UG, Flossmann E, Rothwell PM. Heritability of ischemic stroke in relation to age, vascular risk factors, and subtypes of incident stroke in population-based studies. Stroke 2004:35:819-24.

65 Flossmann E, Rothwell PM. Family history of stroke does not predict risk of stroke after transient ischemic attack. Stroke 2006:37:544-6.

66 Flossmann E, Rothwell PM. Family history of stroke in patients with transient ischemic attack in relation to hypertension and other intermediate phenotypes. Stroke 2005:36:830-5

67 Bevan S, Traylor M, Adib-Samii P, et al. Genetic heritability of ischemic stroke and the contribution of previously reported candidate gene and genomewide associations. Stroke 2012;43:3161-7.

68 Bentley P, Peck G, Smeeth L, et al. Causal relationship of susceptibility genes to ischemic stroke: comparison to ischemic heart disease and biochemical determinants. PLOS ONE 2010;5:e9136.

69 Casas JP, Bautista LE, Smeeth L, et al. Homocysteine and stroke: evidence on a causal link from mendelian randomisation. Lancet 2005;365:224-32.

70 Cheng YC, Anderson CD, Bione S, et al. Are myocardial infarction-associated single-nucleotide polymorphisms associated with ischemic stroke? Stroke 2012;43:980-6.

71 Kannel WB. Risk factors for atherosclerotic cardiovascular outcomes in different arterial territories. J Cardiovasc Risk 1994;1:333-9.

72 Kannel WB, Wolf PA. Peripheral and cerebral atherothrombosis and cardiovascular events in different vascular territories: insights from the Framingham Study. Curr Atheroscler Rep 2006;8:317-23.

73 Casas JP, Hingorani AD, Bautista LE, et al. Meta-analysis of genetic studies in ischemic stroke: thirty-two genes involving approximately 18,000 cases and 58,000 controls. Arch Neurol 2004;61:1652-61.

74 Ariyaratnam R, Casas JP, Whittaker J, et al. Genetics of ischaemic stroke among persons of non-European descent: a meta-analysis of eight genes involving approximately 32,500 individuals. PloS Med 2007:4:e131.

75 Newton-Cheh C, Johnson T, Gateva V, et al. Genome-wide association study identifies eight loci associated with blood pressure. Nat Genet 2009;41:666-76.

76 Ehret GB, Munroe PB, Rice KM, et al. International Consortium for Blood Pressure Genome-Wide Association S. Genetic variants in novel pathways influence blood pressure and cardiovascular disease risk. Nature 2011;478:103-9.

77 Biffi A, Anderson CD, Jagiella JM, et al. APOE genotype and extent of bleeding and outcome in lobar intracerebral haemorrhage: a genetic association study. Lancet Neurol 2011;10:702-9.

78 Ebrahim S, Smith G Davey. Mendelian randomization: can genetic epidemiology help redress the failures of observational epidemiology? Hum Genet 2008;123:15-33.

79 Casas JP, Bautista LE, Smeeth L, et al. Homocysteine and stroke: evidence on a causal link from mendelian randomisation. Lancet 2005;365:224-32.

80 Zhou YH, Tang JY, Wu MJ, et al. Effect of folic acid supplementation on cardiovascular outcomes: a systematic review and meta-analysis. PLOS ONE 2011;6:e25142.

81 Holmes MV, Newcombe P, Hubacek JA, et al. Effect modification by population dietary folate on the association between MTHFR genotype, homocysteine, and stroke risk: a meta-analysis of genetic studies and randomised trials. Lancet 2011;378:584-94.

82 Clarke R, Bennett DA, Parish S, et al. Homocysteine and coronary heart disease: meta-analysis of MTHFR case-control studies, avoiding publication bias. PloS Med 2012;9:e1001177.

83 Burgess S, Thompson SG. Avoiding bias from weak instruments in Mendelian randomization studies. Int J Epidemiol 2011;40:755-64.

84 Lettre G, Rioux JD. Autoimmune diseases: insights from genome-wide association studies. Hum Mol Genet 2008;17:R116-21.

85 Easton DF, Eeles RA. Genome-wide association studies in cancer. Hum Mol Genet 2008;17:R109-15.

86 Gretarsdottir S, Thorleifsson G, Reynisdottir ST, et al. The gene encoding phosphodiesterase 4D confers risk of ischemic stroke. Nat Genet 2003;35:131-8.

87 Rosand J, Bayley N, Rost N, et al. Many hypotheses but no replication for the association between PDE4D and stroke. Nat Genet 2006;38:1091-2; author reply 1092-1093.
88 Bevan S, Porteous L, Sitzer M, et al. Phosphodiesterase 4D gene, ischemic stroke, and asymptomatic carotid atherosclerosis. Stroke 2005;36:949-53.

89 Bevan SDM, Gschwendtner A, Kuhlenbäumer G, et al. Variation in the PDE4D Gene and Ischemic Stroke Risk. A Systematic Review and Metaanalysis on 5200 Cases and 6600 Controls. Stroke 2008:39:1966-71.

90 Matsushita T, Kubo M, Yonemoto K, et al. Lack of association between variations of PDE4D and ischemic stroke in the Japanese population. Stroke 2009:40:1245-51.

91 Holliday EG, Maguire JM, Evans TJ, et al. Common variants at 6p21.1 are associated with large artery atherosclerotic stroke. Nat Genet 2012;44:1147-51.

92 Worrall BB, Mychaleckyj JC. PDE4D and stroke: a real advance or a case of the Emperor's new clothes? Stroke 2006;37:1955-7.

93 Bellenguez C, Bevan S, Gschwendtner A, et al.; International Stroke Genetics C Wellcome Trust Case Control C. Genome-wide association study identifies a variant in HDAC9 associated with large vessel ischemic stroke. Nat Genet 2012;44:328-33.

94 Gudbjartsson DF, Holm H, Gretarsdottir S, et al. A sequence variant in ZFHX3 on $16 q 22$ associates with atrial fibrillation and ischemic stroke. Nat Genet 2009;41:876-8

95 Gudbjartsson DF, Arnar DO, Helgadottir A, et al. Variants conferring risk of atria fibrillation on chromosome 4q25. Nature 2007;448:353-7.

96 Ellinor PT, Lunetta KL, Albert CM, et al. Meta-analysis identifies six new susceptibility loci for atrial fibrillation. Nat Genet 2012;44:670-5.

97 Traylor M, Farrall M, Holliday EG, et al. Genetic risk factors for ischaemic stroke and its subtypes (the METASTROKE Collaboration): a meta-analysis of genome-wide association studies. Lancet Neurol 2012;11:951-62.

98 Lao 0, Lu TT, Nothnagel M, et al. Correlation between genetic and geographic structure in Europe. Curr Biol 2008;18:1241-8.

99 Schrijvers EM, Schurmann B, Koudstaal PJ, et al. Genome-wide association study of vascular dementia. Stroke 2012;43:315-19.

100 Verhaaren BF, de Boer R, Vernooij MW, et al. Replication study of chr17q25 with cerebral white matter lesion volume. Stroke 2011;42:3297-9.

101 Kubo M, Hata J, Ninomiya T, et al. A nonsynonymous SNP in PRKCH (protein kinase $($ eta) increases the risk of cerebral infarction. Nat Genet 2007;39:212-17.

102 Yamada Y, Fuku N, Tanaka M, et al. Identification of CELSR1 as a susceptibility gene for ischemic stroke in Japanese individuals by a genome-wide association study. Atherosclerosis 2009;207:144-9.

103 Hata J, Matsuda K, Ninomiya T, et al. Functional SNP in an Sp1-binding site of AGTRL1 gene is associated with susceptibility to brain infarction. Hum Mol Genet 2007;16:630-9.

104 Matarin M, Brown WM, Singleton A, et al. Whole genome analyses suggest ischemic stroke and heart disease share an association with polymorphisms on chromosome 9p21. Stroke 2008;39:1586-9.

105 Bilguvar K, Yasuno K, Niemela M, et al. Susceptibility loci for intracranial aneurysm in European and Japanese populations. Nat Genet 2008;40:1472-7.

106 Ikram MA, Seshadri S, Bis JC, et al. Genomewide association studies of stroke. N Engl J Med 2009;360:1718-28.

107 Helgadottir A, Manolescu A, Thorleifsson G, et al. The gene encoding 5-lipoxygenase activating protein confers risk of myocardial infarction and stroke. Nat Genet 2004;36:233-9.

108 Debette $\mathrm{S}$, Bis JC, Fornage $\mathrm{M}$, et al. Genome-wide association studies of MRI-defined brain infarcts: meta-analysis from the CHARGE Consortium. Stroke 2010;41:210-17

109 International Stroke Genetics C, Wellcome Trust Case-Control C. Failure to validate association between 12p13 variants and ischemic stroke. N Engl J Med 2010;362:1547-50.

110 Anderson CD, Biffi $A$, Rahman $\mathrm{R}$, et al. Common mitochondrial sequence variants in ischemic stroke. Ann Neurol 2011;69:471-80.

111 DeStefano AL, Atwood LD, Massaro JM, et al. Genome-wide scan for white matter hyperintensity: the Framingham Heart Study. Stroke 2006;37:77-81.

112 Bis JC, Kavousi M, Franceschini N, et al. Meta-analysis of genome-wide association studies from the CHARGE consortium identifies common variants associated with carotid intima media thickness and plaque. Nat Genet 2011:43:940-7.

113 Cotlarciuc, I, Khan, MA, Maheshwari, A, et al. Bio-repository of DNA in stroke: a study protocol of three ancestral populations. J R Soc Med Cardiovasc Dis 2012;1:10.

114 Yadav S, Schanz R, Maheshwari A, et al. Bio-Repository of DNA in stroke (BRAINS): a study protocol. BMC Med Genet 2011;12:34

115 Cole JW, Stine OC, Liu $X$, et al. Rare variants in ischemic stroke: an exome pilot study. PLOS ONE 2012;7:e35591.

116 Matarin M, Simon-Sanchez J, Fung $\mathrm{HC}$, et al. Structural genomic variation in ischemic stroke. Neurogenetics 2008;9:101-8.

117 Tiszlavicz Z, Somogyvari F, Szolnoki Z, et al. Genetic polymorphisms of human beta-defensins in patients with ischemic stroke. Acta Neurol Scand 2012;126:109-15

118 Norskov MS, Frikke-Schmidt R, Loft $\mathrm{S}$, et al. Copy number variation in glutathione S-transferases M1 and T1 and ischemic vascular disease: four studies and meta-analyses. Circ Cardiovasc Genet 2011:4:418-28. 